\title{
RGS10 Regulates the Expression of Cyclooxygenase-2 and Tumor Necrosis Factor Alpha through a G Protein- Independent Mechanism ${ }^{\mathbb{S}}$
}

\author{
Mohammed Alqinyah, Faris Almutairi, Menbere Y. Wendimu, and Shelley B. Hooks
}

Hooks Laboratory, Department of Pharmaceutical and Biomedical Sciences, University of Georgia, Athens, Georgia

Received January 11, 2018; accepted July 11, 2018

\begin{abstract}
The small regulator of $\mathrm{G}$ protein signaling protein RGS10 is a key regulator of neuroinflammation and ovarian cancer cell survival; however, the mechanism for RGS10 function in these cells is unknown and has not been linked to specific $G$ protein pathways. RGS10 is highly enriched in microglia, and loss of RGS10 expression in microglia amplifies production of the inflammatory cytokine tumor necrosis factor $\alpha(\mathrm{TNF} \alpha)$ and enhances microglia-induced neurotoxicity. RGS10 also regulates cell survival and chemoresistance of ovarian cancer cells. Cyclooxygenase-2 (COX-2)-mediated production of prostaglandins such as prostaglandin $\mathrm{E}_{2}\left(\mathrm{PGE}_{2}\right)$ is a key factor in both neuroinflammation and cancer chemoresistance, suggesting it may be involved in RGS10 function in both cell types, but a connection between RGS10 and COX-2 has not been reported. To address these questions, we completed a mechanistic
\end{abstract}

study to characterize RGS10 regulation of TNF $\alpha$ and COX-2 and to determine if these effects are mediated through a $G$ protein-dependent mechanism. Our data show for the first time that loss of RGS10 expression significantly elevates stimulated COX-2 expression and $\mathrm{PGE}_{2}$ production in microglia. Furthermore, the elevated inflammatory signaling resulting from RGS10 loss was not affected by $G \alpha_{i}$ inhibition, and a RGS10 mutant that is unable to bind activated $G$ proteins was as effective as wild type in inhibiting TNF $\alpha$ expression. Similarly, suppression of RGS10 in ovarian cancer cells enhanced TNF $\alpha$ and COX-2 expression, and this effect did not require $G_{i}$ activity. Together, our data strongly indicate that RGS10 inhibits COX-2 expression by a $\mathrm{G}$ protein-independent mechanism to regulate inflammatory signaling in microglia and ovarian cancer cells.

\section{Introduction}

Regulators of G protein signaling (RGS) are a family of proteins that classically act as activators of the intrinsic GTPase activity of heterotrimeric $\mathrm{G} \alpha$ subunits (Watson et al., 1996). Owing to this GTPase-accelerating protein (GAP) activity and inhibition of signaling initiated by $\mathrm{G}$ protein-coupled receptors (GPCRs), RGS proteins play numerous roles in physiologic and pathologic conditions in diverse systems. However, multiple studies have revealed actions of RGS proteins that are independent of GTPase-accelerating activity, recently reviewed in Sethakorn et al. (2010). These noncanonical functions of RGS proteins can affect a variety of targets, including GPCRs, kinases, and transcription factors (Sethakorn et al., 2010). Therefore, to investigate the molecular mechanism of specific RGS protein actions, a critical initial question to answer is whether the RGS protein is acting in a classic GAP-dependent or noncanonical GAP-independent mechanism. The small RGS

Funding for this work was provided by the National Institutes of Health National Institute for Neurologic Disorders and Stroke [Grant NS101161].

https://doi.org/10.1124/mol.118.111674.

S This article has supplemental material available at molpharm. aspetjournals.org. protein RGS10 regulates inflammatory and survival signaling in multiple cell types (Hooks et al., 2010; Lee et al., 2011, 2013), and has been proposed as a potential drug target for neuroinflammatory disease and ovarian cancer. However, the mechanisms by which RGS10 affects inflammatory and survival signaling are undefined, hampering the development of RGS10targeted therapeutic strategies.

RGS10 is the smallest member of the R12 RGS subfamily with no functional domains outside of the RGS domain. RGS10 has been shown to selectively target $\mathrm{G} \alpha_{\mathrm{i}}$ family $\mathrm{G}$ proteins via classic GAP activity (Hunt et al., 1996), and is highly enriched in immune cells, including peripheral macrophages and microglia (Lee et al., 2008, 2013). Loss of RGS10 in microglia amplifies production of inflammatory cytokines, such as tumor necrosis factor $\alpha(\mathrm{TNF} \alpha)$ and interleukin $1 \beta$, and enhances microglia-induced neurotoxicity triggered by the toll-like receptor (TLR) ligand lipopolysaccharide (LPS) (Lee et al., 2011). Reciprocally, activation of microglia by LPS induces epigenetic silencing of RGS10, which we predict serves to amplify inflammatory signaling (Alqinyah et al., 2017). In addition to its anti-inflammatory role in microglia, RGS10 also regulates survival of ovarian cancer cells, and loss of RGS10 induces chemoresistance in ovarian cancer cells

ABBREVIATIONS: $\mathrm{AlF}_{4}^{-}$, aluminum fluoride; COX-2, cyclooxygenase-2; ERK, extracellular signal-regulated kinase; GAP, GTPase-accelerating protein; GAPDH, glyceraldehyde-3-phosphate dehydrogenase; GPCR, G protein-coupled receptor; HRP, horseradish peroxidase; LPA, lysophosphatidic acid; LPS, lipopolysaccharide; $P \mathrm{PE}_{2}$, prostaglandin $\mathrm{E}_{2}$; PTX, pertussis toxin; RGS, regulator of $\mathrm{G}$ protein signaling; siRNA, small interfering RNA; TLR, toll-like receptor; TNF $\alpha$, tumor necrosis factor $\alpha$; WT, wild type. 
(Hooks et al., 2010; Ali et al., 2013). Cyclooxygenase-2 (COX-2)_ mediated production of prostaglandins such as prostaglandin $\mathrm{E}_{2}\left(\mathrm{PGE}_{2}\right)$ is also a key factor in both neuroinflammation and cancer chemoresistance (Minghetti, 2004; Bijman et al., 2008), suggesting that this pathway may be related to RGS10 effect; however, the effect of RGS10 on COX-2 expression and function is not known.

The goal of the current study was to test the hypothesis that RGS10 regulates TNF $\alpha$ and COX-2 through a GAP-dependent mechanism. Our data show for the first time that RGS10 deficiency significantly elevates LPS-stimulated COX-2 expression and release of $\mathrm{PGE}_{2}$ from microglia. Furthermore, the elevated inflammatory signaling resulting from RGS10 loss was not affected by $\mathrm{G} \alpha_{\mathrm{i}}$ inhibition, and a RGS10 GAP-deficient mutant was as effective in inhibiting $\mathrm{TNF} \alpha$ expression as wildtype (WT) RGS10, suggesting that the anti-inflammatory functions of RGS10 are not mediated by its classic GAP activity on $\mathrm{G}$ proteins. We also show for the first time that RGS10 regulates TNF $\alpha$ and COX-2 expression in ovarian cancer cells through a $\mathrm{G}_{\mathrm{i}}$-independent mechanism.

\section{Materials and Methods}

Cells and Reagents. The murine BV-2 microglia cell line was a gift from G. Hasko at the University of Medicine and Dentistry of New Jersey (Newark, NJ) and was previously generated by Blasi et al. (1990). BV-2 cells were maintained in Dulbecco's modified Eagle's medium (VWR, Radnor, PA) supplemented with 10\% fetal bovine serum (Thermo Fisher Scientific, Waltham, MA). The HEK-Blue hTLR4 cell line was purchased from InvivoGen (San Diego, CA) and was maintained in Dulbecco's modified Eagle's medium (VWR) with $10 \%$ fetal bovine serum and HEK-Blue Selection antibiotics (InvivoGen) to selectively maintain cells overexpressing TLR4 and adapter proteins. The human SKOV-3 cell line was purchased from ATCC (Manassas, VA) and was maintained in McCoy's 5A, 1X (Iwakata \& Grace Modification) medium (Corning, Corning, NY) supplemented with L-glutamine and $10 \%$ fetal bovine serum.

Lipopolysaccharide was purchased from Sigma-Aldrich (St. Louis, MO), recombinant mouse CXCL12/SDF-1 alpha protein was purchased from R\&D systems (Minneapolis, MN), pertussis toxin (PTX) was obtained from Tocris (Pittsburgh, PA), and lysophosphatidic acid (LPA) was obtained from Avanti Polar Lipids (Alabaster, AL).

Small Interfering RNA and Plasmid Transfection. Mouse and human small interfering RNA (siRNA) duplexes were purchased from Santa Cruz Biotechnology (Dallas, TX) (mouse: sc-36411, human: sc-36410) and Lipofectamine-LTX with PLUS reagent was purchased from Thermo Fisher Scientific. The mouse siRNA used herein is the same as was described and validated by Lee et al. (2008). The final concentration of siRNA in the culture medium was $60 \mathrm{nM}$, and the transfection was performed according to the manufacturer's protocol. Following transfection, cells were cultured an additional 48 hours in an antibiotic-free culture medium before assessing expression or function. The specificity of knockdown was determined by comparing the expression of RGS10 with RGS2, which is also highly expressed in microglia, and RGS12 and RGS14, which exhibit the highest sequence similarity to RGS10 (Supplemental Fig. 1). Real-time polymerase chain reaction was performed using the following primer sequences: mouse RGS10 forward: 5'-TCCATGACGGAGATGGGAG-3', mouse RGS10 reverse: 5'-AACAAGACATTCTCTTCGCTGAA-3'; mouse RGS2 forward: 5'-GAGAAAATGAAGCGGACACTCT-3', mouse RGS2 reverse: 5'-GCAGCCAGCCCATATTTACTG-3'; mouse RGS12 forward: 5'-GTGACCGTTGATGCTTTCG-3', mouse RGS12 reverse: 5'-ATCGCATGTCCCACTATTCC-3'; and mouse RGS14 forward: 5'-AAATCCCCGCTGTACCAAG-3', mouse RGS14 reverse: 5' GTGACTTCCCAGGCTTCAG-3'.
Ha-tagged RGS10-2 DNA plasmids were purchased from the cDNA Resource Center (Bloomsburg University, Bloomsberg, PA). The E52K mutation was generated with the QuikChange site-directed mutagenesis kit (Stratagene, San Diego, CA) using the following primer sequences: forward: 5'-TA AAA AAG GAA TTC AGT GAA AAA AAT; reverse: 5'-GC TAG CCA AAA CAA AAC ATT TTT TTC A. Mutagenesis resulted in a single nucleotide change from $\mathrm{G} \rightarrow \mathrm{A}$, corresponding to codon GAA $\rightarrow$ AAA, and glutamic acid $\rightarrow$ lysine at position 52 of human RGS10-2

For transfection, $0.5 \mu \mathrm{g}$ of plasmid DNA was used per well of a 24-well plate, or scaled up or down appropriately for different sized wells or plates. DNA plasmids were added to cells with lipofectamine reagent according to the manufacturer's instructions (Thermo Fisher Scientific), and cells were cultured an additional 48 hours in antibiotic-free medium prior to assessing function.

Western Blot Analysis. Cells were lysed in SDS-PAGE sample buffer $(0.5 \mathrm{M}$ Tris $\mathrm{pH} 6.8,10 \%$ SDS, glycerol, $\beta$-mercaptoethanol, bromophenol blue), and the samples were subjected to SDS-PAGE using standard protocols followed by transfer to nitrocellulose membranes. Primary antibodies for RGS10, COX-2, P65, and GNAI3 were purchased from Santa Cruz Biotechnology. Phosphorylated extracellular signal-regulated kinase (ERK), ERK, phospho-P65, P-AKT, and AKT were obtained from Cell Signaling Technology (Danvers, MA), and glyceraldehyde-3-phosphate dehydrogenase (GAPDH) was purchased from Millipore Technologies (Temecula, CA). Following primary antibody incubation, the suitable secondary horseradish peroxidase (HRP)-conjugated antibodies were used to incubate the membranes: donkey anti-goat IgG-HRP (Santa Cruz Biotechnology), goat anti-rabbit IgG-HRP (Millipore Technologies), and goat antimouse IgG-HRP (Bethyl, Montgomery, TX). The membranes were visualized utilizing an enhanced chemiluminescent substrate for detection of HRP (ThermoScientific, Waltham, MA), and quantified using FlourChem HD2 software (Proteinsimple, San Jose, CA). The values were normalized to endogenous control GAPDH.

Quantitative Real-Time Polymerase Chain Reaction. Isolation of mRNA was performed using TRIzol reagent (Invitrogen/Life Technologies, Carlsbad, CA) and cDNA was synthesized using a HighCapacity cDNA Reverse Transcription Kit (Applied Biosystems, Foster City, CA). Actin and/or GAPDH were used as housekeeping genes for normalization. Calculating the fold difference was performed using the $2^{-\Delta \Delta C T}$ method. Mouse actin and COX-2 mouse and human primers were purchased from Sigma-Aldrich. Mouse TNF $\alpha$ primers and human GAPDH and $\mathrm{TNF} \alpha$ primers were obtained from Integrated DNA Technologies (Coralville, IA). The mouse primers used were the following: actin forward 5'-GGC TGT ATT CCC CTC CAT CG-3', actin reverse 5' -CCA GTT GGT AAC AAT GCC ATG T-3'; COX2 forward 5' - TGCAAGATCCACAGCCTACC-3', COX-2 reverse 5'GCTCAGTTGAACGCCTTTTG-3'; and TNF $\alpha$ forward 5 '-CCT GTA GCC CAC GTC GTA G-3', TNF $\alpha$ reverse $5^{\prime}$-GGG AGT AGA CAA GGT ACA ACC C-3' . The human primers used were the following: GAPDH forward, 5'-GCCAAGGTCATCCATGACAACT-3', GAPDH reverse, 5'-GAGGGGCCATCCACAGTCTT-3'; TNF $\alpha$ forward 5'-CTCTTCTGCCTGCACTTTG-3', TNF $\alpha$ reverse 5'-ATGGGCTACAGGCTTGTCACTC-3'; and COX-2 forward 5'-CCCTTGGGTGTCAAAGGTAA-3', COX-2 reverse 5'-GCCCTCGCTTATGATCTGTC-3'.

Coimmunoprecipitation. For the coimmunoprecipitation experiments in BV-2 microglia, cells were plated in $15 \mathrm{~cm}$ dishes and then lysed with $1.5 \mathrm{ml}$ of a modified lysis buffer ( $50 \mathrm{mM}$ Tris-HCl, $150 \mathrm{mM}$ $\mathrm{NaCl}, 6 \mathrm{mM} \mathrm{MgCl} \mathrm{Mg}_{2}, 1 \% \mathrm{NP} 40$ ) containing protease/phosphatase inhibitor cocktail (Cell Signaling Technology). Cell lysate was left for 30 minutes on ice and subsequently centrifuged at $27,216 \mathrm{~g}(15,000 \mathrm{rpm})$ for 10 minutes at $4^{\circ} \mathrm{C}$. Cell lysates were incubated with either GDP $(10 \mu \mathrm{M})$ alone or with $\mathrm{GDP}+\mathrm{AlCl}_{3}(100 \mu \mathrm{m})+\mathrm{NaF}(10 \mathrm{mM})$ at $20^{\circ} \mathrm{C}$ with gentle shaking for 30 minutes. Next, $2 \mu \mathrm{g}$ of $\mathrm{G} \alpha{ }_{\mathrm{i}} 3$ antibody (Santa Cruz Biotechnology) or normal rabbit IgG (Santa Cruz Biotechnology) was added. In the coimmunoprecipitation experiments HEK-hTLR4293 cells were transfected prior to the coimmunoprecipitation with $0.25 \mu \mathrm{g}$ 
of RGS10 WT, RGS10 E52K, and G $\alpha_{\mathrm{i}} 3$. Following transfection, the coimmunoprecipitation was performed as described previously.

$\mathbf{P G E}_{2}$ Measurement. To quantify the levels of $\mathrm{PGE}_{2}$ in culture medium, BV-2 microglia culture medium was collected and centrifuged at $1500 \mathrm{rpm}$ for 10 minutes at $4^{\circ} \mathrm{C}$. Enzyme-linked immunosorbent assay was performed using a $\mathrm{PGE}_{2}$ enzyme-linked immunosorbent assay kit (Enzo, Farmingdale, NY) to measure $\mathrm{PGE}_{2}$ levels in media following the manufacturer's protocol.

CRISPR/Cas RGS10 Knockout. Mouse RGS10 sgRNA CRISPR/ Cas9 all-in-one lentivector (K4107305) (gRNA Sequence: 49 TGTTTTGCAGATATCCATGA) and scrambled control vector (K010) were purchased from ABM Inc. (Richmond, BC, Canada). Vectors (10 ng) were transformed into Proclone competent DH5alpha cells and transformants were selected with $100 \mu \mathrm{g} / \mathrm{ml}$ carbenicillin. All plasmids were isolated and the sequences were confirmed. Recombinant lentiviral particles were produced in 293T cells by packaging $10 \mu \mathrm{g}$ of expression sgRNA CRISPR/Cas9 vectors with $10 \mu \mathrm{g}$ of ABM Inc.'s third generation (LV053) packaging mix according to the company's lentivirus packaging protocol. Lentifectin transfection reagent (G074) was used in the transfection complex to facilitate DNA uptake by $293 \mathrm{~T}$ cells. Transfection media were removed after 24 hours and replaced with complete growth media for an additional 24 hours. Viral media were harvested 48 hours after transfection and centrifuged at $3000 \mathrm{rpm}$ for 15 minutes at $4^{\circ} \mathrm{C}$ to pellet cell debris. Cleared supernatant was then filtered with a low-protein binding $0.45 \mu \mathrm{M}$ sterile filter and further concentrated by centrifugation at 25,000 rpm for 2 hours at $4^{\circ} \mathrm{C}(\sim 120,000 \mathrm{~g}$; SW28 rotor, Beckman, Brea, CA). Viral pellets were resuspended and stored at $-80^{\circ} \mathrm{C}$ until transfection. Different volumes of prepared lentivirus were transfected into BV-2 cells in the presence of polybrene $(0.8 \mu \mathrm{g} / \mathrm{ml})$. Media containing polybrene were replaced with complete culture media after 24 hours. Forty-eight hours after transfection, cells were split and transfectants were selected for stable expression using $6 \mu \mathrm{g} / \mathrm{ml}$ puromycin selection media, as determined by a kill curve. Genome editing was then assayed by western blotting and sequencing.

Experimental Rigor and Statistical Analysis. All quantitative real-time polymerase chain reaction data presented were performed in experimental and technical triplicates, except where indicated in the figure legends. Technical triplicates for each experiment were averaged prior to statistical analysis. All western images were quantified using densitometry of raw, unmodified image data, including background subtraction. Some images of western data have been modified slightly to adjust brightness, as indicated in the figure legends. To analyze the data for statistical differences between groups, data were analyzed using analysis of variance, followed by Tukey's multiple comparisons tests. The statistical significance cutoff ranges indicated in the figures correspond to: $* P<0.05$, ${ }^{* *} P<0.01$, and ${ }^{* * *} P<0.001$.

\section{Results}

RGS10 Knockdown Elevates LPS-Induced Produc-

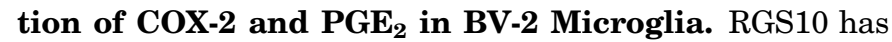
been shown to regulate expression of multiple inflammatory mediators following activation with LPS, but an effect on COX-2 has not been reported. Due to the essential roles of COX-2 in inflammation in microglia, we aimed to test whether LPS-stimulated COX-2 is also affected by RGS10 loss. We treated BV-2 microglia with RGS10-targeted siRNA or control siRNA for 24 hours before stimulating with LPS (10 ng/ml) for an additional 24 hours. Transient transfection of siRNA duplexes in BV-2 cells consistently resulted in 50\%-70\% reduction of RGS10 mRNA and protein levels, with no effect on mRNA expression of RGS2, a widely expressed RGS with high expression in microglia, or on mRNA expression of RGS12 and RGS14, which have the highest sequence similarity to RGS10 (Supplemental Fig. 1). As we have previously reported (Alqinyah et al., 2017), activation of microglia with
LPS for 24 hours also suppressed RGS10 expression, and this suppression was additive with siRNA-mediated RGS10 suppression (Fig. 1C). RGS10 knockdown significantly enhanced LPS-induced upregulation of COX-2 mRNA (Fig. 1A). Similarly, LPS treatment resulted in higher levels of COX-2 protein in BV-2 cells, and this effect was significantly enhanced in cells transfected with RGS10 siRNA compared with control siRNA cells, suggesting that endogenous RGS10 suppresses COX-2 protein expression in BV-2 microglia (Fig. 1, B and C). One of the essential functions of COX-2 is the production of prostaglandins including $\mathrm{PGE}_{2}$, which is a critical mediator of neuroinflammation (Andreasson, 2010). Therefore, we tested the effect of RGS10 loss on LPS-induced production of $\mathrm{PGE}_{2}$ in BV-2 microglia. RGS10 knockdown enhanced the LPS-stimulated release of $\mathrm{PGE}_{2}$ into microglia culture medium by approximately $25 \%$ (Fig. 1D). Finally, to validate the results observed following transient siRNAmediated knockdown, we generated BV-2 stable RGS10 knockout cells using CRISPR/Cas9 as described in Materials and Methods. Complete suppression of RGS10 expression resulted in robust amplification of LPS-stimulated COX-2 mRNA and protein levels (Fig. 1, E-G) Taken together, the results indicate that endogenous RGS10 regulates the expression of COX-2 and suppresses $\mathrm{PGE}_{2}$ release from microglia following LPS stimulation.

RGS10 Acts as a Classic GTPase-Activating Protein in Microglia, but Does Not Affect TLR4-Induced Acute Signaling. RGS10 deactivates $\mathrm{G} \alpha_{\mathrm{i}}$ proteins, and signaling crosstalk has been reported between $\mathrm{G} \alpha \alpha_{\mathrm{i}}$ pathways and LPSstimulated TLRs (Fan et al., 2007; Marty and Ye, 2010). Thus, we sought to determine whether the ability of RGS10 to regulate LPS-stimulated inflammatory signaling in microglia is mediated by RGS10 regulation of $\mathrm{G} \alpha_{\mathrm{i}}$. First, we determined if LPS stimulation of BV-2 cells impacted association of RGS10 with $\mathrm{G} \alpha$ subunits in BV-2 cells. BV-2 cells were treated with LPS $(10 \mathrm{ng} / \mathrm{ml})$ for 3 hours, and then cell lysates were immunoprecipitated with $\mathrm{G} \alpha_{\mathrm{i}}, \mathrm{G} \alpha_{\mathrm{q}}$, or control antibodies and the associated proteins were immunoblotted for the $\mathrm{G}$ proteins and RGS10. Multiple studies have described strong $\mathrm{G} \alpha_{\mathrm{i}}$ selectivity of the RGS10 RGS domain (Ajit and Young, 2005; Taylor et al., 2016); however, it has also been reported that RGS10 can display weak interactions with $\mathrm{G} \alpha_{\mathrm{q}}$ (Soundararajan et al., 2008). Our results show that LPS activation of BV-2 cells enhances RGS10 association with $\mathrm{G} \alpha_{\mathrm{i}}$, but no detectable association with $\mathrm{G} \alpha_{\mathrm{q}}$ was observed (Fig. 2A). Next, we aimed to confirm that RGS10 expressed in BV-2 microglia can act as a $\mathrm{G} \alpha_{\mathrm{i}}$ GAP by assessing binding between RGS10 and transition state $\mathrm{G} \alpha_{\mathrm{i}}$. This conformation of $\mathrm{G} \alpha$ subunits is the high-affinity substrate for RGS domain GAP activity and is mimicked and stabilized in the presence of GDP with aluminum fluoride $\left(\mathrm{AlF}_{4}^{-}\right.$), while $\mathrm{G} \alpha$-GDP has low affinity for RGS domains (Berman et al., 1996; Tesmer et al., 1997). BV-2 cell lysates were immunoprecipitated with $\mathrm{G} \alpha_{\mathrm{i}} 3$ or $\mathrm{G} \alpha_{\mathrm{q}}$ targeted antibody or control IgG in the presence of GDP or GDP plus $\mathrm{AlF}_{4}^{-}$. Our results revealed a strong and distinct binding between RGS10 and $\mathrm{G} \alpha_{\mathrm{i}} 3-\mathrm{AlF}_{4}^{-}$, with no detectable binding between RGS10 and $\mathrm{G} \alpha_{\mathrm{i}} 3$-GDP or between RGS10 and either form of $\mathrm{G} \alpha_{\mathrm{q}}$ (Fig. 1B). Therefore, consistent with the literature, endogenous RGS10 appears to function as a $\mathrm{G}_{\mathrm{i}}$-selective GAP in BV-2 microglia.

GPCRs and TLRs both activate ERK and AKT kinase cascades as well as the transcription factor $\mathrm{NF}_{\kappa} \mathrm{B}$, and these pathways facilitate signaling crosstalk between the two 
A

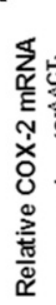

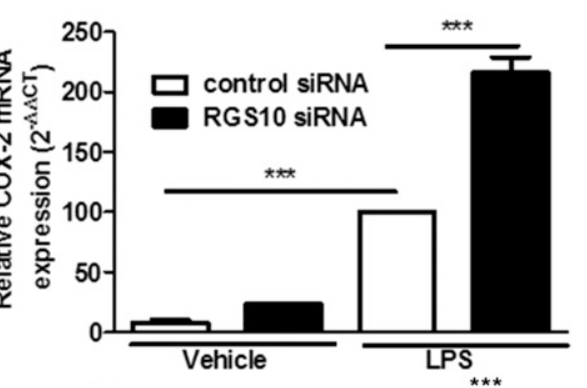

C

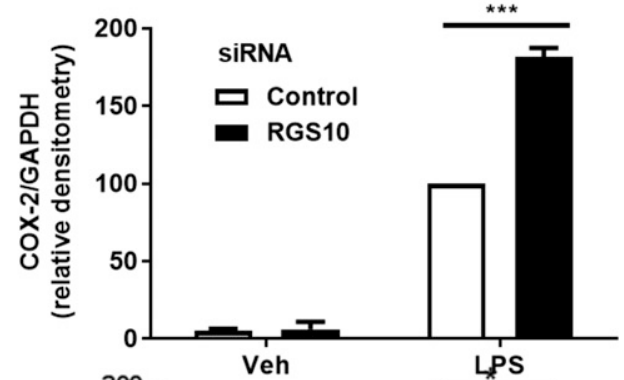

D

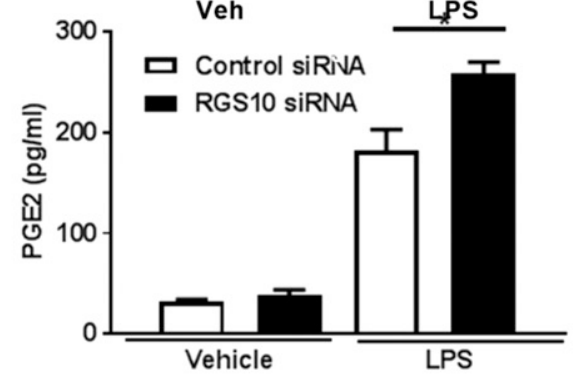

E

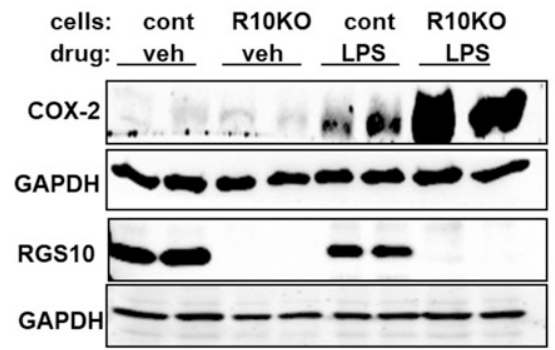

G

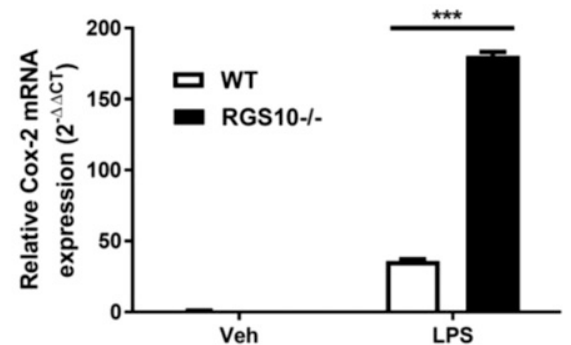

B
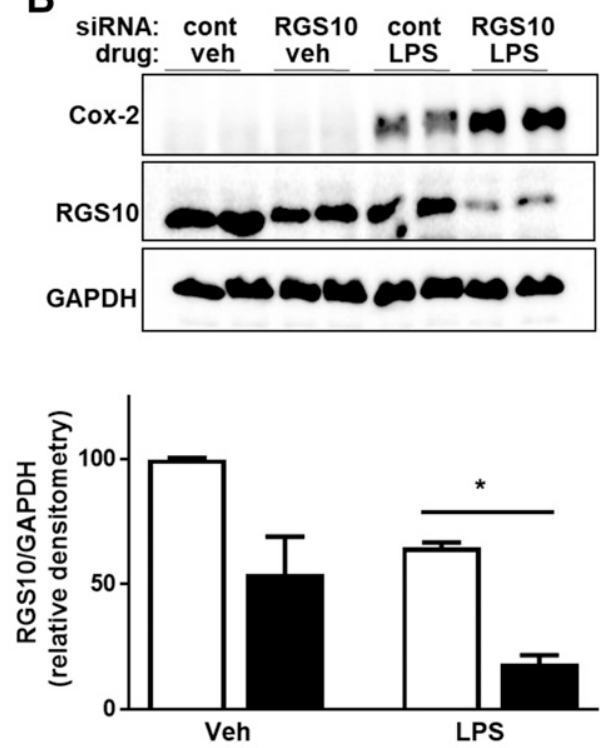

F
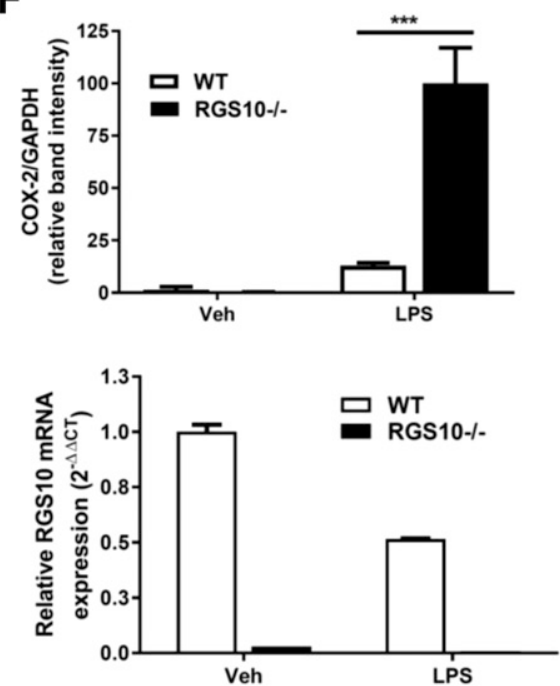

Fig. 1. Loss of RGS10 enhances LPS-stimulated COX-2 expression and PGE 2 production in BV-2 microglia. (A) BV-2 microglia cells were plated in sixwell plates and simultaneously transfected with either control or RGS10 siRNA. Cells were cultured for 24 hours and then incubated with vehicle or LPS $(10 \mathrm{ng} / \mathrm{ml})$ for an additional 24 hours. RNA extraction, cDNA synthesis, and quantitative real-time polymerase chain reaction were performed as described in Materials and Methods. Expression of COX-2 mRNA was normalized to the control actin, and relative expression levels were calculated by the $2^{-\Delta \Delta \mathrm{Ct}}$ method. Data were analyzed from two independent experiments. The difference between groups was analyzed by analysis of variance (ANOVA), followed by Tukey's test. Data are presented as mean \pm S.E.M., where $* P<0.05, * * P<0.01$, and $* * * P<0.001$. (B and C) BV-2 microglia were plated in 24-well plates and transfected with control or RGS10 siRNA for 24 hours and then treated with vehicle or LPS for an additional 24 hours. Cells were lysed, and SDS-PAGE was performed followed by immunoblotting using specific antibodies against COX-2, RGS10, and the loading control GAPDH. (B) The image is representative of two independent experiments. (C) Images from two independent western experiments were quantified with densitometry. (D) BV-2 microglia cells were plated in six-well plates and simultaneously transfected with either control or RGS10 siRNA. Cells were

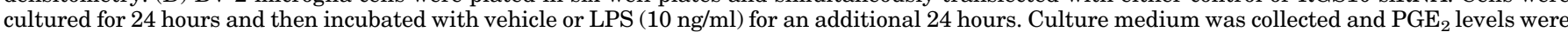
measured using enzyme-linked immunosorbent assay. Conversion of raw absorbance values to picograms per milliliter concentration was conducted using a standard curve following the manufacturer's protocol. Data were analyzed from four independent experiments and the difference between groups was analyzed by ANOVA, followed by Tukey's test. Data are presented as mean \pm S.E.M., where $* P<0.05$. (E-G) BV-2 cells were stably infected with control or RGS10-targeted CRISPR/Cas9 lentivirus as described in Materials and Methods. Control and RGS10 knockout cells were treated with $10 \mathrm{ng} / \mathrm{ml}$ 
A

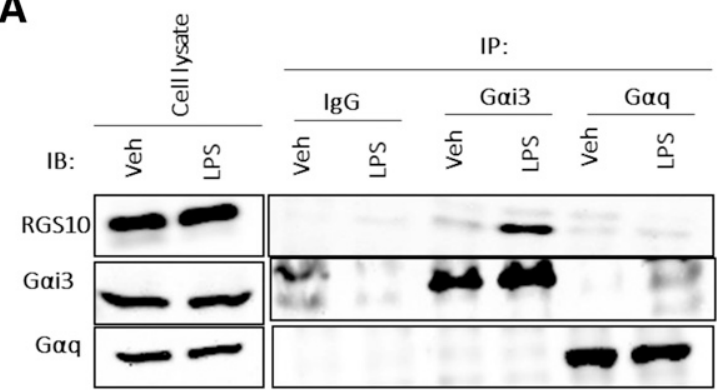

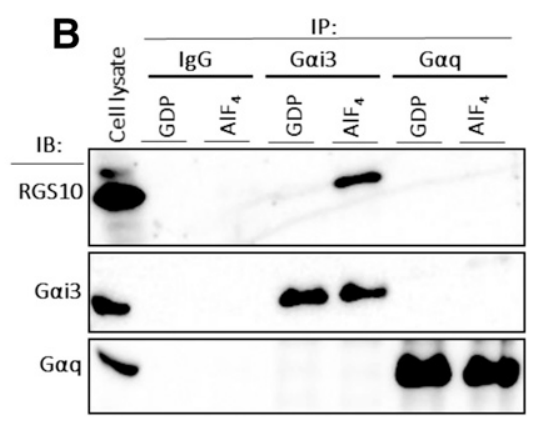

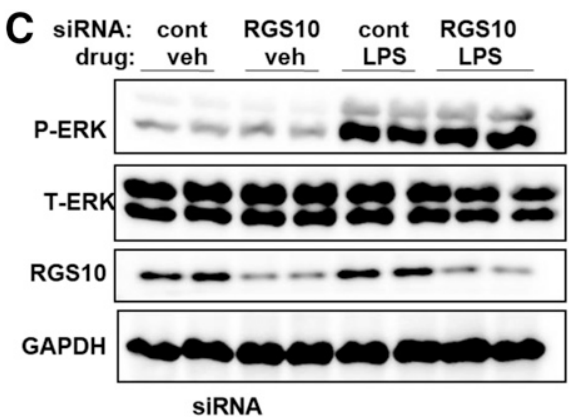

SIRNA

E

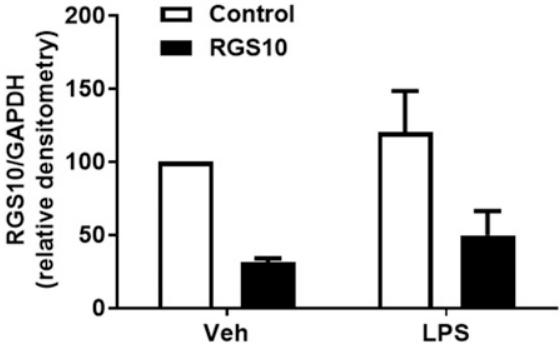

D siRnA: cont RGS10 cont RGS10

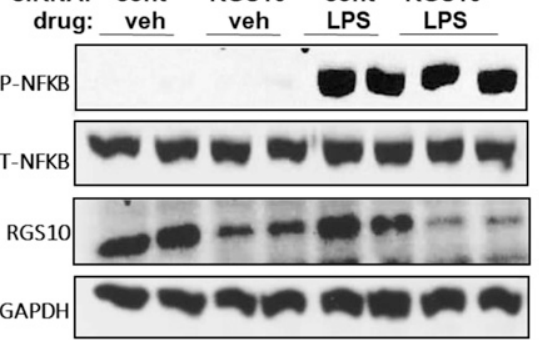

GAPDH
Fig. 2. Endogenous RGS10 associates with the active form of $\mathrm{G} \alpha$, but does not regulate ERK or $\mathrm{NF}_{\kappa} \mathrm{B}$ phosphorylation. (A) BV-microglia cells were plated in $15 \mathrm{~cm}$ dishes and treated with vehicle or $10 \mathrm{ng} / \mathrm{ml}$ LPS for 3 hours and then lysed using modified radioimmunoprecipitation assay lysis buffer as described in Materials and Methods. Coimmunoprecipitation was performed using $\mathrm{G} \alpha_{\mathrm{i}} 3, \mathrm{G} \alpha_{\mathrm{q}}$, or control IgG antibodies. Western blot analysis was conducted to probe for RGS10, $\mathrm{G} \alpha_{\mathrm{i}} 3$, or $\mathrm{G} \alpha_{\mathrm{q}}$. (B) BV-2 cell lysates were incubated with $\operatorname{GDP}(10 \mu \mathrm{M})$ alone or with $\mathrm{AlF}_{4}^{-}$for 30 minutes at $25^{\circ} \mathrm{C}$ and immunoprecipitated as previously described. (C and D) BV-2 microglia cells were plated in 24-well plates and transfected with control or RGS10 siRNA before incubation with vehicle or LPS for 20 minutes. Cells were lysed, and SDS-PAGE was performed followed by immunoblotting using specific antibodies against (C) phosphoERK, total ERK, RGS10, and the loading control GAPDH, and (D) phospho-P65 $\mathrm{NF}_{\kappa} \mathrm{B}$, total $\mathrm{P} 65 \mathrm{NF}_{\kappa} \mathrm{B}$, RGS10 and GAPDH. (E) Quantification of RGS10 protein levels in experiments shown in $(\mathrm{C}$ and D) was performed using densitometry. Combined data from two independent experiments were analyzed. (A-D) Images are representative of two independent experiments. receptor classes and downstream inflammatory signaling (Ye, 2001; Fan et al., 2004; Dauphinee et al., 2011). Given the ability of RGS10 to deactivate $\mathrm{G} \alpha_{\mathrm{i}}$ and the ability of $\mathrm{G} \alpha_{\mathrm{i}}$ pathways to activate $\mathrm{ERK}, \mathrm{AKT}$, and $\mathrm{NF} \kappa \mathrm{B}$, we predicted that RGS10 regulation of TLR4-induced COX-2 and $\mathrm{PGE}_{2}$ production may be mediated by RGS10 deactivation of $\mathrm{G} \alpha_{\mathrm{i}}$ proteins that impact the ability of TLR4 to initiate these pathways. To explore this hypothesis further, we tested LPSinduced phosphorylation of p42/44 ERK with or without RGS10 knockdown. RGS10 knockdown had no effect on ERK phosphorylation following 20 minutes of LPS treatment (Fig. 2C). Similarly, acute LPS treatment stimulated phosphorylation of AKT and p38 in BV-2 microglia, but RGS10 knockdown had no effect on these responses (data not shown). Phosphorylation of $\mathrm{NF}_{\kappa} \mathrm{B}$ subunit p65, an early event in the $\mathrm{NF} \kappa \mathrm{B}$ activation cascade, was also significantly enhanced by LPS treatment, but this response was not affected by RGS10 knockdown (Fig. 2D). Therefore, endogenous RGS10 appears to function as a $\mathrm{G} \alpha_{\mathrm{i}}$ GAP, but does not regulate acute LPSstimulated signaling pathways that are coregulated by $\mathrm{G} \alpha_{\mathrm{i}}$.

The Effect of $\mathbf{G} \alpha_{i}$ Inhibition on RGS10 KnockdownInduced Enhancement of TLR4 Signaling. To further explore the role of $\mathrm{G} \alpha_{\mathrm{i}}$ in RGS10's effects on inflammatory signaling, we next sought to determine whether enhanced
COX-2 expression resulting from RGS10 knockdown is the result of amplified $\mathrm{G} \alpha_{\mathrm{i}}$ signaling. If the primary mechanism by which RGS10 regulates LPS-stimulated inflammatory signaling is through regulation of $\mathrm{G} \alpha_{\mathrm{i}}$ GAP activity, then the effects of RGS10 knockdown should be reversed by inhibition of $\mathrm{G} \alpha_{\mathrm{i}}$. To test this prediction, we measured the expression of COX-2 mRNA after LPS treatment and RGS10 knockdown with or without pretreatment with the $\mathrm{G}_{\mathrm{i} / \mathrm{o}}$ family inhibitor PTX. Surprisingly, the RGS10 siRNA-mediated increase in LPSstimulated COX-2 mRNA production (Fig. 3A) or protein levels (Fig. 3B) was completely resistant to PTX pretreatment. Since RGS10 loss has also been shown to enhance the production of the inflammatory cytokine $\mathrm{TNF} \alpha$, we further tested whether RGS10 knockdown-induced increase in $\mathrm{TNF} \alpha$ expression was sensitive to $\mathrm{G} \alpha_{\mathrm{i}}$ inhibition. Like COX-2, the increase in LPS-stimulated $\mathrm{TNF} \alpha$ expression mediated by RGS10 knockdown was not affected by PTX (Fig. 3C). To ensure that the dose of PTX used is fully efficacious in BV-2 cells, we confirmed that PTX completely blocked CXCL12stimulated AKT phosphorylation, which has been shown to be mediated by $\mathrm{G} \alpha_{\mathrm{i}}$ signaling (Kumar et al., 2012) (Fig. 3D). Collectively, these data suggest that RGS10 knockdownmediated amplification of COX-2 and $\mathrm{TNF} \alpha$ expression is not mediated by enhanced $\mathrm{G} \alpha_{\mathrm{i}}$ signaling. 


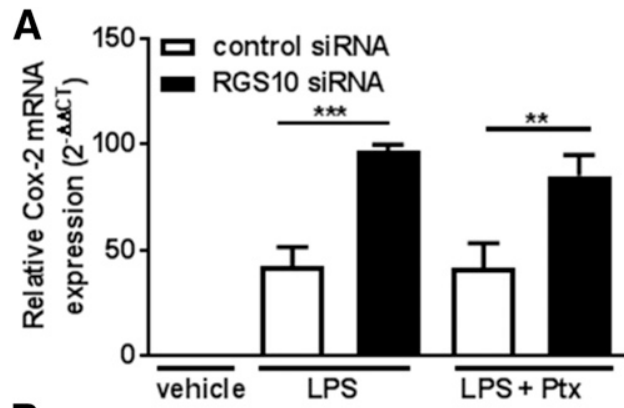

B
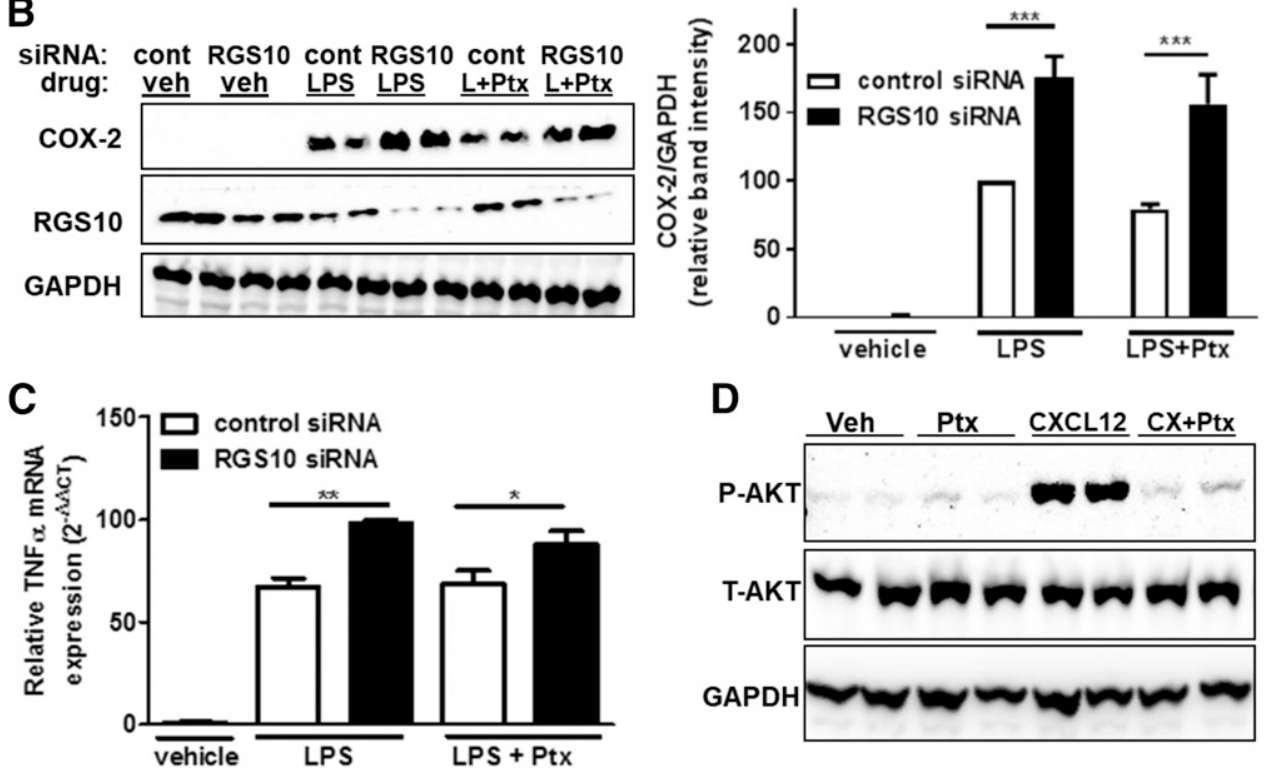

Fig. 3. PTX pretreatment does not affect RGS10 knockdown-induced increase of LPS-stimulated COX-2 and TNF $\alpha$. (A) BV-2 microglia cells were plated in six-well plates and transfected with either control or RGS10 siRNA. Twenty-four hours after transfection, cells were incubated with vehicle or LPS $(10 \mathrm{ng} / \mathrm{ml})$ for 24 hours with or without PTX $(100 \mathrm{ng} / \mathrm{ml})$. PTX was added 4 hours prior to LPS and included throughout the 24-hour LPS treatment. Expression of COX-2 mRNA was normalized to the endogenous control actin, and relative expression was calculated by the $2^{-\Delta \Delta \mathrm{Ct}}$ method. Data were analyzed from four independent experiments and the difference between groups was analyzed by analysis of variance (ANOVA), followed by Tukey's test. Data are presented as mean \pm S.E.M., where ${ }^{*} P<0.05$, $* * P<0.01$, and $* * * P<0.001$. (B) BV-2 microglia cells were plated in 24 -well plates and transfected with control or RGS10 siRNA. Twenty-four hours after transfection, cells were treated with vehicle or LPS for an additional 24 hours with or without PTX $(100 \mathrm{ng} / \mathrm{ml})$. PTX was added 4 hours prior to LPS and was included throughout the 24-hour LPS treatment. Cells were lysed, and SDS-PAGE was performed followed by immunoblotting using specific antibodies against COX-2, RGS10, and the loading control GAPDH. Band intensity was analyzed from three independent experiments and the difference between groups was analyzed by ANOVA, followed by Tukey's test. Data are presented as mean \pm S.E.M., where $* P<0.05, * * P<0.01$, and $* * * P<0.001$. (C) BV-2 microglia cells were plated in six-well plates and transfected with either control or RGS10 siRNA. After 24 hours, cells were incubated with vehicle or LPS (10 ng/ml) for an additional 24 hours with or without PTX (100 ng/ml). PTX was added 4 hours prior to LPS and was included throughout the 24-hour LPS treatment. Expression of TNF $\alpha$ mRNA was normalized to the endogenous control actin, and relative expression was calculated by the $2^{-\Delta \Delta \mathrm{Ct}}$ method. Data were analyzed from five independent experiments and the difference between groups was analyzed by ANOVA, followed by Tukey's test. Data are presented as mean \pm S.E.M., where $* P<0.05$, $* * P<0.01$, and ***P $P<0.001$. (D) BV-2 microglia were plated in 24-well plates and then treated with vehicle or CXCL12 (200 ng/ml) for 5 minutes with or without preincubation with PTX $(100 \mathrm{ng} / \mathrm{ml})$ overnight. Following incubation, cells were lysed, and SDS-PAGE was performed followed by immunoblotting using specific antibodies against phospho-AKT, total AKT, and GAPDH. The graph is representative of two independent experiments.

Evaluating HEK293-HourTLR4 Cells as a Model to Study the Effect of RGS10 on TLR4-Induced Inflammatory Signaling. To more directly test the possibility that RGS10 regulates LPS-stimulated inflammatory signaling through a GAP-independent mechanism, we sought to assess whether RGS10 G protein binding activity is required for this effect by comparing the effects of exogenous WT and GAPdeficient RGS10. BV-2 microglia cells are not an appropriate model for this experiment due to their low transfection efficiency and high levels of endogenous RGS10. As an alternative, we used HEK293-hourTLR4 cells because they are easy to transfect, express low endogenous RGS10 levels, and stably overexpress TLR4 receptors. To confirm that this cell line is responsive to LPS treatment, we treated HEK293hourTLR4 cells with LPS $(10 \mathrm{ng} / \mathrm{ml})$, a dose that potently activates TLR4 signaling in BV-2 microglia. LPS caused a dramatic increase in TNF $\alpha$ mRNA levels (Fig. 4A), and phosphorylation of $\mathrm{p} 65-\mathrm{NF} \kappa \mathrm{B}$ and $\mathrm{p} 44 / 42$ mitogen-activated protein kinase (data not shown) in HEK293-hourTLR4 cells. However, COX-2 expression was not affected by LPS treatment in these cells, demonstrating that not all downstream TLR4 signaling pathways observed in BV-2 microglia will be recapitulated in HEK293-hourTLR4 cells. Nonetheless, the cells provided a suitable platform to assess the effect of exogenous RGS10 on LPS-induced production of $\mathrm{TNF} \alpha$. To address this question, we overexpressed WT RGS10 via transient plasmid transfection, inducing robust expression of exogenous RGS10 mRNA and protein. RGS10 overexpression suppressed LPS-induced production of TNF $\alpha$ after 24 hours of LPS treatment (Fig. 4A). In contrast, RGS10 overexpression 
A

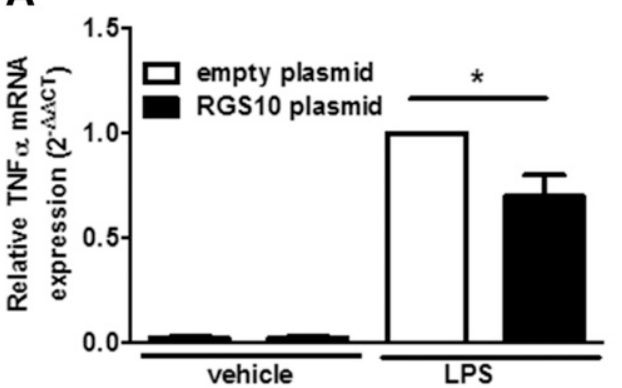

B

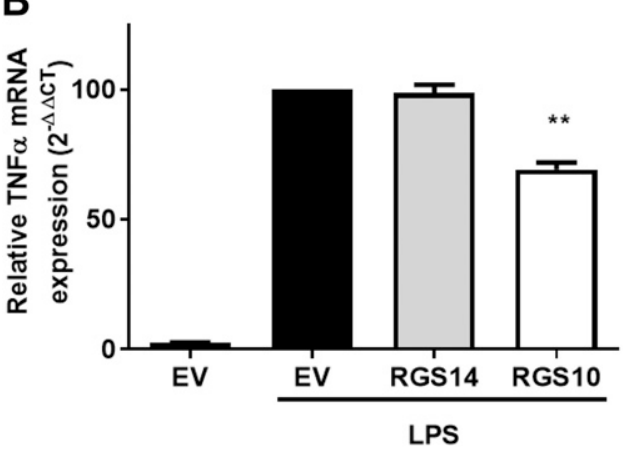

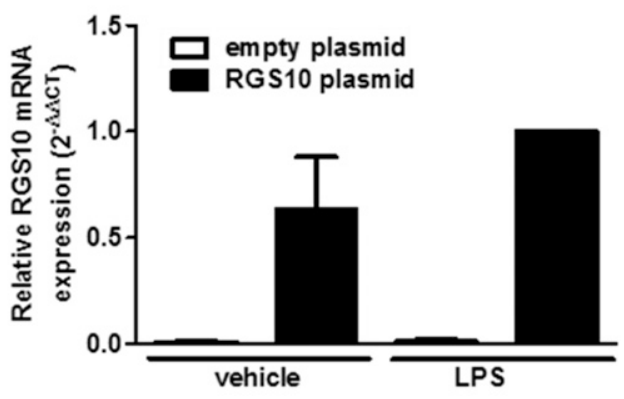

Fig. 4. HEK293-hourTLR4 cells express $\mathrm{TNF} \alpha$ in response LPS and express high levels of exogenous RGS10. (A) HEK293hourTLR4 cells were plated in 24-wells plate and allowed to reach $\sim 80 \%$ confluency. Cells were transfected with either empty vector or RGS10 plasmid for 48 hours and treated with vehicle or LPS 24 hours after transfection. mRNA expression of TNF $\alpha$ and RGS10 was normalized to the endogenous control actin, and relative expression was calculated by the $2^{-\Delta \Delta \mathrm{Ct}}$ method. (B). HEK293hourTLR4 cells were transfected with empty vector or plasmid encoding RGS10 or the RGS domain of RGS14. RNA was isolated and $\mathrm{TNF} \alpha$ expression was assessed as previously described. Data were analyzed from three independent experiments, each with technical duplicates, and the difference between groups was analyzed by analysis of variance, followed by Tukey's test. Data are presented as mean \pm S.E.M., where $* P<$ 0.05 and $* * P<0.01$ had no effect on acute LPS-induced activation of ERK and NF $\kappa \mathrm{B}$ (data not shown). Finally, to determine if this effect is shared among closely related RGS domains, we overexpressed the RGS domain of RGS14 and compared the effect on LPS-stimulated TNF $\alpha$ expression. The exogenous RGS14 RGS domain, when expressed at similar levels to exogenous RGS10, did not have any effect on LPS-stimulated TNF $\alpha$ expression (Fig. 4B). These results suggest that RGS10 regulates TLR4-stimulated gene expression to a greater extent than homologous RGS domains.

The Effect of RGS10 WT and E52K Mutant on TLR4Induced Signaling in HEK293-HourTLR4 Cells. We generated a single amino acid mutation E52K in RGS10 corresponding to a previously characterized GAP-dead mutation in the RGS domain of RGS12 (Sambi et al., 2006). To confirm that the mutation did not affect expression or protein stability, we compared RGS10 protein levels 48 hours after transient transfection with plasmid encoding RGS10 WT or RGS10 E52K in HEK293-hourTLR4 cells. Our results indicate that the RGS10E52K mutant was expressed equally to the WT (Fig. 5A). To confirm that the RGS10 E52K mutant is indeed unable to bind the active form of $\mathrm{G}$ proteins, we transfected HEK293-hourTLR4 cells with WT and E52K RGS10 and performed coimmunoprecipitation to compare their $\mathrm{G}$ protein binding abilities. Only RGS10WT, and not the E52K mutant, was able to bind active $\mathrm{G} \alpha_{\mathrm{i}} 3-\mathrm{GDP}-\mathrm{AlF}_{4}^{-}$(Fig. 5B), which confirms that the RGS10 E52K mutant has deficient GAP activity. Next, we aimed to confirm that the RGS10 GAPdeficient mutant lost the ability to suppress $\mathrm{G}_{\mathrm{i}}$-mediated signaling pathways. Unfortunately, comparing the effect of overexpressed WT and mutant RGS10 on GPCR-evoked $\mathrm{G}_{\mathrm{i}}$ signaling in HEK293-hourTLR4 cells was not possible because we have not observed any effect of exogenous WT RGS10 overexpression on signaling stimulated by specific $\mathrm{G}_{\mathrm{i}}$-coupled GPCRs, including the multiple purinergic, lysophospholipid, and chemokine receptor agonists that we tested (data not shown). It is possible that RGS10 regulates $\mathrm{G}_{\mathrm{i}}$-mediated signaling downstream from select GPCRs that we have not yet tested. However, it is also possible that, although RGS10 can interact with activated $\mathrm{G}_{\mathrm{i}}$ in cell lysates, it does not function to regulate acute signaling following activation of cell-surface GPCRs. To indirectly address the effect of the EK mutant on signaling, we turned to the SKOV-3 cells, in which we have shown that exogenous RGS10 overexpression regulates basal ERK mitogen-activated protein kinase activity in the presence of serum. In these cells, overexpression of WT RGS10, but not EK mutant RGS10, suppressed ERK phosphorylation (Fig. 5C), suggesting that this mutant is defective in regulating $\mathrm{G}$ protein signaling pathways.

Finally, we compared the ability of WT and GAP-dead E52K RGS10 to suppress LPS-induced TNF $\alpha$ mRNA in HEK293hourTLR 4 cells. In sharp contrast to the lack of $\mathrm{G} \alpha_{\mathrm{i}}$ interaction activity of RGS10 E52K, we show that overexpression of both RGS10 WT and E52K mutant resulted in similar inhibition of LPS-induced production of TNF $\alpha$ (Fig. 5D). RGS10 mRNA was measured in parallel with $\mathrm{TNF} \alpha$ in each experiment to ensure equivalent transfection efficiency of RGS10WT and E52K. These data provide further compelling evidence that RGS10 inhibits TLR4 signaling in a mechanism independent of its $G$ protein interaction and GTPase accelerating activity.

Loss of RGS10 Enhances TNF $\alpha$ and COX-2 Expression in Ovarian Cancer Cells, and This Effect Is Mediated via a G $\alpha_{\mathbf{i}}$-Independent Mechanism. The previous data demonstrate RGS10 suppression of the inflammatory cytokine $\mathrm{TNF} \alpha$ and the inflammatory enzyme COX-2 in microglia. However, RGS10 has also been shown to play important roles in other physiologic systems, most notably in ovarian cancer cells (Hooks et al., 2010; Ali et al., 2013). RGS10 suppresses survival signaling and maintains sensitivity to chemotherapeutic-induced cell death in these cells, but the mechanism is unknown and has not been directly linked to a $\mathrm{G} \alpha_{\mathrm{i}}$-mediated pathway. Inflammatory signaling including COX-2- and TNF $\alpha$-mediated pathways are directly linked to ovarian cancer chemoresistance (Symowicz et al., 2005; Gu et al., 2008). Based on our previous results demonstrating that 
A
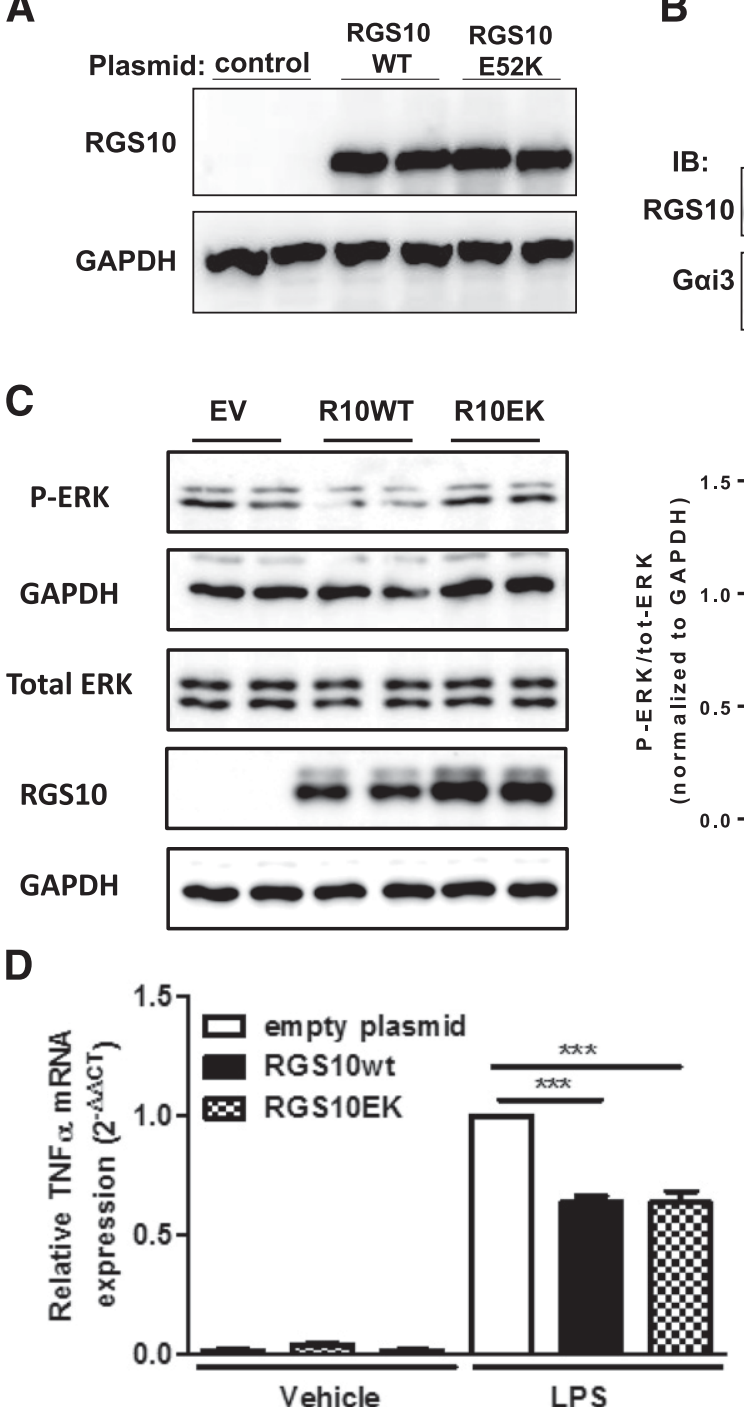

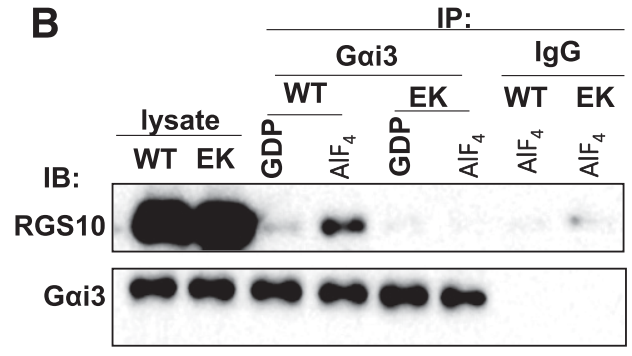
E52K RGS10 equally suppress LPS-induced TNF $\alpha$ expression. (A) HEK293-hourTLR4 cells were plated in 24-well plates and allowed to reach approximately $80 \%$ confluency before transfection with $0.5 \mu \mathrm{g}$ of RGS10 WT or RGS10 E52K plasmids. Forty-eight hours after transfection, cells were lysed, and SDS-PAGE was performed followed by immunoblotting using specific antibodies against RGS10 and GAPDH. (B) HEK293-hourTLR4 cells were plated in $10 \mathrm{~cm}$ dishes and transfected with pcDNA encoding $\mathrm{G} \alpha_{\mathrm{i}} 3$ along with either RGS10 WT or RGS10 E52K plasmids. Forty-eight hours after transfection, cells were lysed with modified lysis buffer and cell lysates were incubated with GDP $(10 \mu \mathrm{M})$ alone or with $\mathrm{AlF}_{4}^{-}$. Coimmunoprecipitation was performed using $\mathrm{G} \alpha \alpha_{\mathrm{i}} 3$ or control IgG antibodies. Western blot analysis was conducted to probe for RGS10 and $\mathrm{G} \alpha{ }_{\mathrm{i}} 3$. (C) SKOV-3 cells were transfected with empty vector or plasmid encoding WT RGS10 or E52K RGS10. Cell lysates were analyzed using SDS-PAGE and western blotting for phosphorylated p42/p44 ERK mitogen-activated protein (Map) kinase and total p42/p44 ERK Map kinase. Each Map kinase signal was normalized to GAPDH, and the ratio of phosphorylated/total Map kinase was calculated. (D) HEK293-hourTLR4 cells were plated in 24-well plates and transfected with RGS10 WT or RGS10 E52K plasmids for 48 hours. Cells were incubated with LPS $(10 \mathrm{ng} / \mathrm{ml})$ for 24 hours. mRNA expression of TNF $\alpha$ was normalized to the endogenous control actin, and relative expression was calculated by the $2^{-\Delta \Delta \mathrm{Ct}}$ method. (A and B) Images are representative of two independent experiments. (C and D) Data were analyzed from two independent experiments, each with technical duplicates, and the difference between groups was analyzed by analysis of variance, followed by Tukey's test. Data are presented as mean \pm S.E.M., where $* P<$ $0.05, * * P<0.01$, and $* * * P<0.001$. n.s., not significant.
RGS10 regulates the expression of both COX-2 and $\mathrm{TNF} \alpha$ in microglia, we aimed to test whether RGS10 also regulates inflammatory mediators in ovarian cancer, and if so whether RGS10 also functions in a G protein-independent mechanism in ovarian cancer cells. We transfected SKOV-3 ovarian cancer cells with RGS10 siRNA or control siRNA in the presence or absence of PTX, and assessed the mRNA levels of COX-2 and $\mathrm{TNF} \alpha$. SKOV-3 cells transfected with RGS10 siRNA produced significantly higher levels of COX-2 (Fig. 6A) and TNF $\alpha$ (Fig. $6 \mathrm{~B})$ mRNA compared with cells transfected with control siRNA. SKOV-3 cells did not require receptor stimulation to observe an effect of RGS10 knockdown on COX-2 or TNF $\alpha$ expression, and these cells did not respond to LPS treatment with enhanced COX-2 or TNF $\alpha$ expression. As observed in microglia, PTX treatment had no effect on RGS10 siRNA-mediated upregulation of COX-2 and TNF $\alpha$ expression. Successful knockdown of RGS10 following siRNA transfection in SKOV-3 cells was confirmed by real-time polymerase chain reaction (Fig. 6C), and we confirmed that the dose of PTX used in the experiment was sufficient to fully inhibit LPA-mediated ERK phosphorylation in SKOV-3, an established $\mathrm{G} \alpha_{\mathrm{i}}$-mediated event (Fig. 6D) (Hurst et al., 2008). These results demonstrate for the first time that RGS10 regulates inflammatory signaling pathways in ovarian cancer cells and that RGS10 anti-inflammatory actions and mechanisms are not exclusive to immune cells but extend to other models. These findings provide new insight into understanding the mechanisms by which RGS10 suppresses chemoresistance and survival of ovarian cancer cells.

\section{Discussion}

RGS10 regulates cellular physiology and fundamental signaling pathways in microglia (Lee et al., 2008, 2011), macrophages (Lee et al., 2013), T-lymphocytes (Lee et al., 2016), neurons (Lee et al., 2012), osteoclasts (Yang and Li, 2007; Yang et al., 2007, 2013), cardiomyocytes (Miao et al., 2016), platelets (Hensch et al., 2016, 2017), and cancer cells (Hooks et al., 2010; Ali et al., 2013; Cacan et al., 2014; Hooks and Murph, 2015). However, despite its small size and seemingly simple function as a G protein GAP, the molecular mechanisms accounting for RGS10 effects have not been defined. The results presented here significantly expand our understanding of the scope and mechanism of RGS10 regulation of inflammatory signaling. First, we show for the first 
A

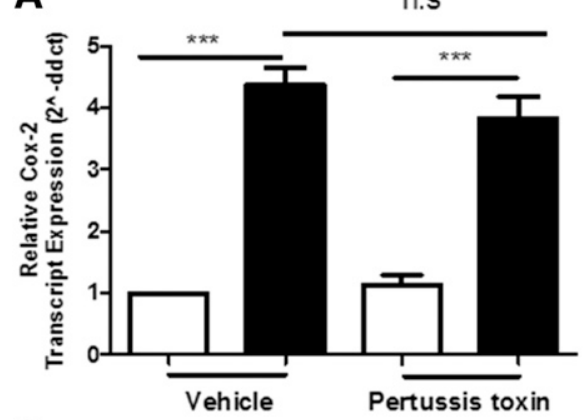

c

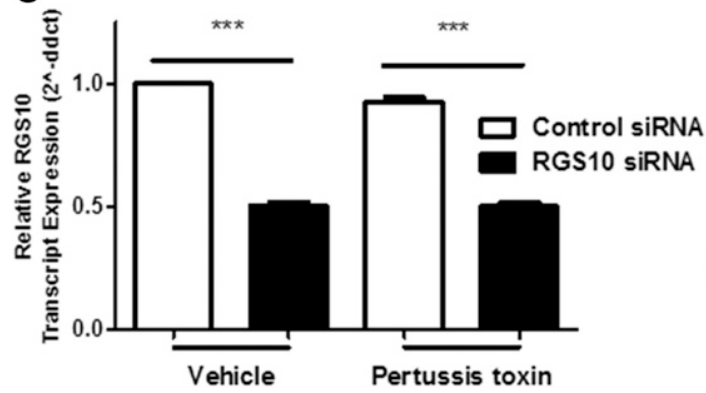

B

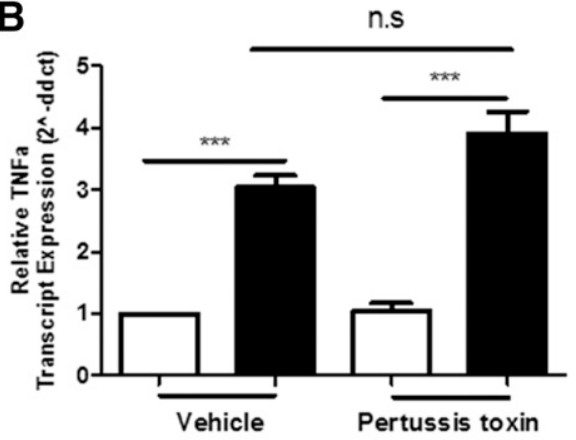

D

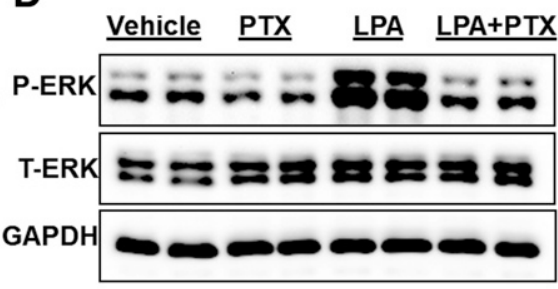

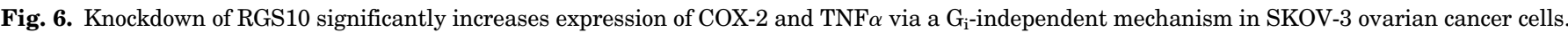

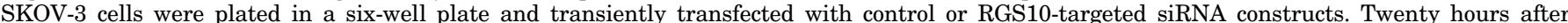

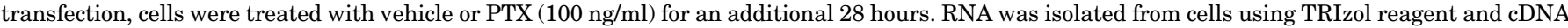

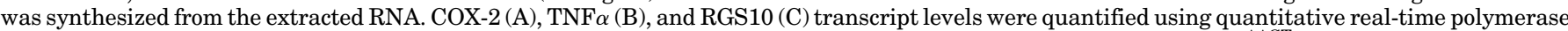

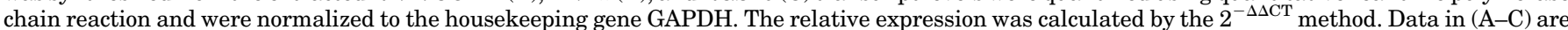

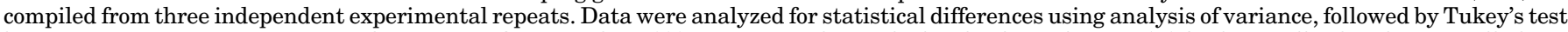

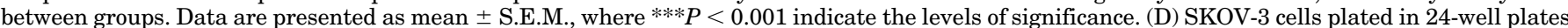

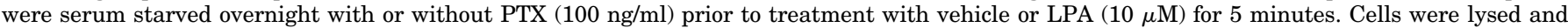

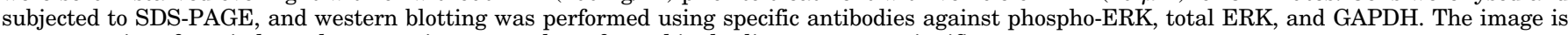
representative of two independent experiments, each performed in duplicate. n.s., not significant.

time that RGS10 regulates COX-2 expression and subsequent prostaglandin production. Given the essential role of COX-2 and $\mathrm{PGE}_{2}$ in the physiology of diverse systems, this finding expands the potential relevance of RGS10 in physiology and disease. Furthermore, our results provide the first evidence of regulation of inflammatory signaling pathways in cancer cells by RGS10, establishing a potential explanation for the effect of RGS10 on ovarian cancer cell survival. Finally, and most importantly, our results demonstrate that the effect of RGS10 on inflammatory signaling in both microglia and ovarian cancer cells cannot be explained by the ability of RGS10 to suppress $\mathrm{G} \alpha_{\mathrm{i}}$ signaling. We further show that exogenously expressed GAP-deficient RGS10 suppresses LPS-stimulated TNF $\alpha$ to the same extent as WT RGS10. This surprising result suggests novel RGS10 mechanisms and potentially novel binding partners linking RGS10 to inflammatory signaling mediators $\mathrm{TNF} \alpha$ and COX-2.

RGS10 is highly expressed in microglia and RGS10 has profound effects on microglial function. Microglia cells are resident macrophages of the central nervous system and normally function to eliminate pathogens and debris (Kettenmann et al., 2011). However, chronic activation of these cells leads to amplified production of inflammatory cytokines, prostaglandins, and other neurotoxic molecules, ultimately resulting in significant neuronal death and neurodegenerative diseases (Lull and Block, 2010). Microglial RGS10 suppresses the release of TNF $\alpha$ and other inflammatory cytokines following inflammatory triggers (Lee et al.,
2008) and plays a protective role against microglia-induced neurodegeneration of dopaminergic neurons (Lee et al., 2011). Reciprocally, microglial activation by either LPS or TNF $\alpha$ suppresses the expression of RGS10 and reduces RGS10 protein levels by approximately 50\%-70\% for approximately 48 hours (Alqinyah et al., 2017). Thus, endogenous RGS10 silencing mechanisms likely serve to amplify and enhance inflammation in a feed-forward mechanism that sustains a continuous cycle of RGS10 suppression and enhanced production of inflammatory cytokines (Lee et al., 2008; Alqinyah et al., 2017). Importantly, the magnitude and duration of RGS10 suppression induced by transient siRNA transfection recapitulates that observed during suppression of RGS10 expression induced by endogenous activation of microglia (Alqinyah et al., 2017), suggesting that the effects reported here following siRNA knockdown will likely serve to amplify the magnitude of COX-2 and $\mathrm{PGE}_{2}$ signaling during endogenous microglial activation.

COX-2 enhances neuroinflammation and is implicated in microglia-induced neurotoxicity. To further assess inflammatory pathways regulated by RGS10 in microglia, we tested the effect of RGS10 loss on the expression of COX-2 and production of $\mathrm{PGE}_{2}$. RGS10 knockdown enhanced the LPS-induced production of COX-2 and the subsequent release of $\mathrm{PGE}_{2}$ from BV-2 microglia. These findings confirm previous reports demonstrating the anti-inflammatory roles of RGS10 in microglia, and further expand the scope of this effect. COX-2 and $\mathrm{PGE}_{2}$ have been shown to enhance neurodegeneration of dopaminergic neurons 
(Teismann et al., 2003; Sánchez-Pernaute et al., 2004). Therefore, it is possible that the effect of RGS10 on COX-2 and $\mathrm{PGE}_{2}$ production accounts, fully or partially, for the neuroprotective properties of RGS10 on dopaminergic neurons (Lee et al., 2008). Furthermore, since COX-2 and $\mathrm{PGE}_{2}$ play diverse roles in the central nervous system, it is likely that RGS10 serves additional functions in the central nervous system related to COX-2 regulation.

Remarkably, the mechanisms by which RGS10 exerts its anti-inflammatory and neuroprotective actions have not been defined. RGS proteins classically function to accelerate the inactivation of heterotrimeric $\mathrm{G}$ proteins, thereby inhibiting $\mathrm{G}$ protein-mediated signaling pathways (Watson et al., 1996). Indeed, RGS10 has been shown to function as a bona fide GAP with specificity to $\mathrm{G} \alpha_{\mathrm{i}}$ subunits (Hunt et al., 1996), and our data confirm that endogenous RGS10 in microglia does bind transition state $\mathrm{G} \alpha_{\mathrm{i}}$, consistent with its classic GAP function. $\mathrm{G} \alpha_{\mathrm{i}}$ has been shown to enhance LPS-induced activation of multiple acute signaling pathways, including AKT and ERK kinase cascades (Fan et al., 2004; Dauphinee et al., 2011), as well as $\mathrm{NF} \kappa \mathrm{B}$ signaling, which is an essential pathway regulating the production of inflammatory cytokines (Ye, 2001). We also show that LPS stimulation of BV-2 cells enhances RGS10 interaction with $\mathrm{G} \alpha_{\mathrm{i}}$. Therefore, our initial hypothesis was that RGS10, via its classic GTPase-accelerating activity on $\mathrm{G} \alpha_{\mathrm{i}}$, would suppress these acute signaling pathways following LPS treatment. However, RGS10 knockdown had no effect on LPS-induced acute activation of ERK, AKT, or $\mathrm{NF}_{\kappa} \mathrm{B}$. This suggested the possibility that RGS10 may function in a mechanism that is independent of its effect on $\mathrm{G} \alpha \alpha_{\mathrm{i}}$.

Our data also revealed that the amplified TNF $\alpha$ and COX-2 production observed with RGS10 knockdown was not altered by $\mathrm{G} \alpha_{\mathrm{i}}$ inhibition with PTX. While PTX is an established and commonly used inhibitor of $\mathrm{G} \alpha_{\mathrm{i}}$, it can produce $\mathrm{G}$ proteinindependent effects (Mangmool and Kurose, 2011). To more rigorously assess the role of $\mathrm{G} \alpha_{\mathrm{i}}$ in RGS10 function, we created a RGS10 mutant E52K construct based on a previously characterized GAP-dead RGS12 construct (Sambi et al., 2006). Overexpression studies comparing RGS10 E52K and WT RGS10 were performed in HEK293-hourTLR4 cells, which stably express TLR4, MD-2, and CD14 co-receptor genes to recapitulate functional TLR4 signaling. Indeed, we confirmed that HEK293-hourTLR4 cells show dramatically enhanced $\mathrm{TNF} \alpha$ expression and activation of $\mathrm{NF} \kappa \mathrm{B}$ and ERK phosphorylation in response to LPS treatment with similar dose response as BV-2 cells. We demonstrated that overexpression of GAP-deficient mutant RGS10 suppressed LPS-induced TNF $\alpha$ to the same degree as WT, suggesting that $\mathrm{G} \alpha_{\mathrm{i}}$ interaction is not required for the ability of RGS10 to inhibit TLR4 signaling. These data, combined with the observation that RGS10 regulation of LPS-stimulated TNF $\alpha$ and COX-2 is PTX insensitive in BV-2 microglia cells, strongly suggest that RGS10 anti-inflammatory effects are mediated by $\mathrm{G}$ protein-independent mechanisms. Furthermore, we have validated the RGS10E52K mutant as a valuable tool to assess the role of RGS10 GAP activity in mediating the overall functions of RGS10.

In addition to its anti-inflammatory effects in microglia and macrophages, RGS10 has also been shown to regulate ovarian cancer cell survival and chemosensitivity. We have previously postulated that the ability of RGS10 to regulate cell survival in ovarian cancer cells was based on its ability to regulate signaling through $\mathrm{G}_{\mathrm{i}}$-coupled receptors for growth factors such as LPA, a well-established autocrine survival factor that is upregulated in ovarian cancer (Hurst et al., 2008). However, while RGS10 suppression enhanced cell survival and basal AKT signaling, it did not enhance LPA-stimulated AKT or ERK signaling in our previous studies (Hooks et al., 2010). Our current observations demonstrate that RGS10 suppression strongly enhances basal TNF $\alpha$ and COX-2 expression in SKOV-3 ovarian cancer cells, indicating that RGS10 suppresses the production of inflammatory mediators in both immune and nonimmune cells. As observed in BV-2 microglia, $\mathrm{G} \alpha_{\mathrm{i}}$ inhibition had no effect on RGS10 knockdowninduced upregulation of COX-2 and TNF $\alpha$ in SKOV-3. TNF $\alpha$ and COX-2 are strongly implicated in the development of chemoresistance and control of cell survival of many cancers including ovarian cancer, suggesting that these pathways may mediate the effect of RGS10 on chemoresistance (Kulbe et al., 2007; Greenhough et al., 2009). Further studies are warranted to determine if the ability of RGS10 to regulate inflammatory signaling in cancer cells fully accounts for its effects on chemoresistance, and if this mechanism extends to other cancer types.

The notion of GAP-independent mechanisms for RGS proteins is not new, and has been described in multiple RGS family members (see the review in Sethakorn et al., 2010). However, many of these RGS proteins possess, in addition to RGS domains, additional domains that mediate some of these GAP-independent functions. For example, RGS12 and RGS14, the RGS family members most closely related to RGS10, interact with RAS and MEK2 proteins via RBD and PDZ domains, respectively, to perform multiple GAP-independent functions (Willard et al., 2007). In contrast, RGS10 contains only the conserved RGS domain and short, disordered $\mathrm{N}$ - and C-terminal extensions containing sites for regulatory modifications, but no defined functions. This suggests that the RGS domain itself may mediate the GAP-independent function of RGS10. Indeed, G protein-independent interactions and associations have been mapped to the RGS domains of multiple RGS proteins (Popov et al., 2000; Nguyen et al., 2009; Sethakorn et al., 2010), supporting the notion that the RGS domain is much more than just a GAP for G proteins. Structural studies have identified modest differences in the structure and flexibility of the RGS10 RGS domain, compared with other family members, which may allow distinct binding interactions (Soundararajan et al., 2008). Therefore, we predict that $G$ protein-independent mechanisms and binding partners facilitate a significant subset of RGS10 functions. Identification and delineation of these novel molecular mechanisms is a critical next step in understanding RGS10 function, and will facilitate strategic targeting of RGS10 in the diverse pathologies in which its function is implicated.

\section{Acknowledgments}

We thank Dr. Jillian Hurst for assistance in generating the E52K RGS10 mutation.

\section{Authorship Contributions}

Participated in research design: Alqinyah, Hooks. Conducted experiments: Alqinyah, Almutairi, Wendimu. Performed data analysis: Alqinyah, Almutairi, Wendimu, Hooks. Wrote or contributed to the writing of the manuscript: Alqinyah, Hooks. 


\section{References}

Ajit SK and Young KH (2005) Analysis of chimeric RGS proteins in yeast for the functional evaluation of protein domains and their potential use in drug target validation. Cell Signal 17:817-825.

Ali MW, Cacan E, Liu Y, Pierce JY, Creasman WT, Murph MM, Govindarajan R, Eblen ST, Greer SF, and Hooks SB (2013) Transcriptional suppression, DNA methylation, and histone deacetylation of the regulator of G-protein signaling 10 (RGS10) gene in ovarian cancer cells. PLoS One 8:e60185.

Alqinyah M, Maganti N, Ali MW, Yadav R, Gao M, Cacan E, Weng HR, Greer SF, and Hooks SB (2017) Regulator of G protein signaling 10 (Rgs10) expression is transcriptionally silenced in activated microglia by histone deacetylase activity. Mol Pharmacol 91:197-207.

Andreasson K (2010) Emerging roles of $\mathrm{PGE}_{2}$ receptors in models of neurological disease. Prostaglandins Other Lipid Mediat 91:104-112.

Berman DM, Kozasa T, and Gilman AG (1996) The GTPase-activating protein RGS4 stabilizes the transition state for nucleotide hydrolysis. J Biol Chem 271 27209-27212.

Bijman MN, Hermelink CA, van Berkel MP, Laan AC, Janmaat ML, Peters GJ, and Boven E (2008) Interaction between celecoxib and docetaxel or cisplatin in human cell lines of ovarian cancer and colon cancer is independent of COX-2 expression levels. Biochem Pharmacol 75:427-437.

Blasi E, Barluzzi R, Bocchini V, Mazzolla R, and Bistoni F (1990) Immortalization of murine microglial cells by a v-raf/v-myc carrying retrovirus. $J$ Neuroimmunol 27:229-237.

Cacan E, Ali MW, Boyd NH, Hooks SB, and Greer SF (2014) Inhibition of HDAC1 and DNMT1 modulate RGS10 expression and decrease ovarian cancer chemoresistance. PLoS One 9:e87455.

Dauphinee SM, Voelcker V, Tebaykina Z, Wong F, and Karsan A (2011) Heterotrimeric $\mathrm{G}_{\mathrm{i}} / \mathrm{G}_{\mathrm{o}}$ proteins modulate endothelial TLR signaling independent of the MyD88-dependent pathway. Am J Physiol Heart Circ Physiol 301: H2246-H2253.

Fan H, Luttrell LM, Tempel GE, Senn JJ, Halushka PV, and Cook JA (2007) $\beta$-Arrestins 1 and 2 differentially regulate LPS-induced signaling and proinflammatory gene expression. Mol Immunol 44:3092-3099.

Fan H, Peck OM, Tempel GE, Halushka PV, and Cook JA (2004) Toll-like receptor 4 coupled GI protein signaling pathways regulate extracellular signal-regulated kinase phosphorylation and AP-1 activation independent of $\mathrm{NF} \kappa \mathrm{B}$ activation. Shock 22:57-62.

Greenhough A, Smartt HJ, Moore AE, Roberts HR, Williams AC, Paraskeva C, and Kaidi A (2009) The COX-2/PGE 2 pathway: key roles in the hallmarks of cancer and adaptation to the tumour microenvironment. Carcinogenesis 30:377-386.

Gu P, Su Y, Guo S, Teng L, Xu Y, Qi J, Gong H, and Cai Y (2008) Over-expression of COX-2 induces human ovarian cancer cells (CAOV-3) viability, migration and proliferation in association with PI3-k/Akt activation. Cancer Invest 26:822-829.

Hensch NR, Karim ZA, Druey KM, Tansey MG, and Khasawneh FT (2016) RGS10 negatively regulates platelet activation and thrombogenesis. PLoS One 11: e0165984.

Hensch NR, Karim ZA, Qasim H, and Khasawneh FT (2017) RGS10 serves as a braking system for platelet hyperactivity. FASEB J 31 (Supple 1):674.6.

Hooks SB, Callihan P, Altman MK, Hurst JH, Ali MW, and Murph MM (2010) Regulators of G-protein signaling RGS10 and RGS17 regulate chemoresistance in ovarian cancer cells. Mol Cancer 9:289.

Hooks SB and Murph MM (2015) Cellular deficiency in the RGS10 protein facilitates chemoresistant ovarian cancer. Future Med Chem 7:1483-1489.

Hunt TW, Fields TA, Casey PJ, and Peralta EG (1996) RGS10 is a selective activator of $\mathrm{G} \alpha$, GTPase activity. Nature 383:175-177.

Hurst JH, Henkel PA, Brown AL, and Hooks SB (2008) Endogenous RGS proteins attenuate $\mathrm{G} \alpha \alpha_{\mathrm{i}}$-mediated lysophosphatidic acid signaling pathways in ovarian cancer cells. Cell Signal 20:381-389.

Kettenmann H, Hanisch UK, Noda M, and Verkhratsky A (2011) Physiology of microglia. Physiol Rev 91:461-553.

Kulbe H, Thompson R, Wilson JL, Robinson S, Hagemann T, Fatah R, Gould D, Ayhan A, and Balkwill F (2007) The inflammatory cytokine tumor necrosis factor- $\alpha$ generates an autocrine tumor-promoting network in epithelial ovarian cancer cells. Cancer Res 67:585-592.

Kumar R, Tripathi V, Ahmad M, Nath N, Mir RA, Chauhan SS, and Luthra K (2012) CXCR7 mediated Gi $\alpha$ independent activation of ERK and Akt promotes cell survival and chemotaxis in T cells. Cell Immunol 272:230-241.

Lee JK, Chung J, Druey KM, and Tansey MG (2012) RGS10 exerts a neuroprotective role through the PKA/c-AMP response-element (CREB) pathway in dopaminergic neuron-like cells. J Neurochem 122:333-343.

Lee JK, Chung J, Kannarkat GT, and Tansey MG (2013) Critical role of regulator G-protein signaling 10 (RGS10) in modulating macrophage M1/M2 activation. PLoS One 8:e81785.
Lee JK, Chung J, McAlpine FE, and Tansey MG (2011) Regulator of G-protein signaling-10 negatively regulates NF- $\mathrm{B}$ in microglia and neuroprotects dopaminergic neurons in hemiparkinsonian rats. J Neurosci 31:11879-11888.

Lee JK, Kannarkat GT, Chung J, Joon Lee H, Graham KL, and Tansey MG (2016) RGS10 deficiency ameliorates the severity of disease in experimental autoimmune encephalomyelitis. J Neuroinflammation 13:24.

Lee JK, McCoy MK, Harms AS, Ruhn KA, Gold SJ, and Tansey MG (2008) Regulator of G-protein signaling 10 promotes dopaminergic neuron survival via regulation of the microglial inflammatory response. J Neurosci 28:8517-8528.

Lull ME and Block ML (2010) Microglial activation and chronic neurodegeneration. Neurotherapeutics 7:354-365.

Mangmool S and Kurose $\mathrm{H}(2011) \mathrm{G}_{\mathrm{i} / \mathrm{o}}$ protein-dependent and -independent actions of pertussis toxin (PTX). Toxins (Basel) 3:884-899.

Marty C and Ye RD (2010) Heterotrimeric G protein signaling outside the realm of seven transmembrane domain receptors. Mol Pharmacol 78:12-18.

Miao R, Lu Y, Xing X, Li Y, Huang Z, Zhong H, Huang Y, Chen AF, Tang X, Li H, et al. (2016) Regulator of G-protein signaling 10 negatively regulates cardiac remodeling by blocking mitogen-activated protein kinase-extracellular signalregulated protein kinase 1/2 signaling. Hypertension 67:86-98.

Minghetti L (2004) Cyclooxygenase-2 (COX-2) in inflammatory and degenerative brain diseases. J Neuropathol Exp Neurol 63:901-910.

Nguyen CH, Ming H, Zhao P, Hugendubler L, Gros R, Kimball SR, and Chidiac P (2009) Translational control by RGS2. J Cell Biol 186:755-765.

Popov SG, Krishna UM, Falck JR, and Wilkie TM (2000) $\mathrm{Ca}^{2+} /$ calmodulin reverses phosphatidylinositol 3,4,5-trisphosphate-dependent inhibition of regulators of $\mathrm{G}$ protein-signaling GTPase-activating protein activity. J Biol Chem 275:18962-18968.

Sambi BS, Hains MD, Waters CM, Connell MC, Willard FS, Kimple AJ, Pyne S, Siderovski DP, and Pyne NJ (2006) The effect of RGS12 on PDGF $\beta$ receptor signalling to p42/p44 mitogen activated protein kinase in mammalian cells. Cell Signal 18:971-981.

Sánchez-Pernaute R, Ferree A, Cooper O, Yu M, Brownell AL, and Isacson O (2004) Selective COX-2 inhibition prevents progressive dopamine neuron degeneration in a rat model of Parkinson's disease. J Neuroinflammation 1:6.

Sethakorn N, Yau DM, and Dulin NO (2010) Non-canonical functions of RGS proteins. Cell Signal 22:1274-1281.

Soundararajan M, Willard FS, Kimple AJ, Turnbull AP, Ball LJ, Schoch GA, Gileadi C, Fedorov OY, Dowler EF, Higman VA, et al. (2008) Structural diversity in the RGS domain and its interaction with heterotrimeric G protein $\alpha$-subunits. Proc Natl Acad Sci USA 105:6457-6462.

Symowicz J, Adley BP, Woo MM, Auersperg N, Hudson LG, and Stack MS (2005) Cyclooxygenase-2 functions as a downstream mediator of lysophosphatidic acid to promote aggressive behavior in ovarian carcinoma cells. Cancer Res 65:2234-2242.

Taylor VG, Bommarito PA, and Tesmer JJ (2016) Structure of the regulator of G Protein signaling 8 (RGS8)-G $\alpha_{\mathrm{q}}$ complex: molecular basis for $\mathrm{G} \alpha$ selectivity. J Biol Chem 291:5138-5145.

Teismann P, Tieu K, Choi DK, Wu DC, Naini A, Hunot S, Vila M, Jackson-Lewis V, and Przedborski S (2003) Cyclooxygenase-2 is instrumental in Parkinson's disease neurodegeneration. Proc Natl Acad Sci USA 100:5473-5478.

Tesmer JJ, Berman DM, Gilman AG, and Sprang SR (1997) Structure of RGS4 bound to $\mathrm{AlF}_{4}^{-}$-activated $\mathrm{G}_{\mathrm{i} \alpha 1}$ : stabilization of the transition state for GTP hydrolysis. Cell 89:251-261.

Watson N, Linder ME, Druey KM, Kehrl JH, and Blumer KJ (1996) RGS family members: GTPase-activating proteins for heterotrimeric G-protein $\alpha$-subunits. Nature 383:172-175.

Willard MD, Willard FS, Li X, Cappell SD, Snider WD, and Siderovski DP (2007) Selective role for RGS12 as a Ras/Raf/MEK scaffold in nerve growth factormediated differentiation. EMBO $J$ 26:2029-2040.

Yang S, Chen W, Stashenko P, and Li YP (2007) Specificity of RGS10A as a key component in the RANKL signaling mechanism for osteoclast differentiation. $J$ Cell Sci 120:3362-3371.

Yang S, Hao L, McConnell M, Zhou X, Wang M, Zhang Y, Mountz JD, Reddy M, Eleazer PD, Li YP, et al. (2013) Inhibition of Rgs10 expression prevents immune cell infiltration in bacteria-induced inflammatory lesions and osteoclast-mediated bone destruction. Bone Res 1:267-281.

Yang S and Li YP (2007) RGS10-null mutation impairs osteoclast differentiation resulting from the loss of $\left[\mathrm{Ca}^{2+}\right]_{\mathrm{i}}$ oscillation regulation. Genes Dev 21:1803-1816.

Ye RD (2001) Regulation of nuclear factor $\kappa \mathrm{B}$ activation by G-protein-coupled receptors. J Leukoc Biol 70:839-848.

Address correspondence to: Shelley B. Hooks, University of Georgia, 240 West Green Street, Pharmacy South, Athens, GA 30602. E-mail: shooks@ uga.edu 